\title{
THE ORDER TOPOLOGY IN A BICOMPACTLY GENERATED LATTICE
}

\author{
D. C. KENT and C. R. ATHERTON \\ (Received 28 November 1966)
}

\section{Introduction}

The concept of a compactly generated lattice has been studied extensively in connection with decomposition theory (see [1]). This paper investigates the order topology in a lattice which is, along with its dual, compactly generated (hence, bicompactly generated). We show that order convergence is topological and that the order topology is Hausdorff, totally disconnected, and has an open subbase of ideals and dual ideals in any bicompactly generated lattice; furthermore, with an additional restriction, the lattice operations are continuous in the order topology. Next we consider the order topology in certain special types of compactly generated lattices, namely atomic Boolean algebras and sub-complete lattices of atomic Boolean algebras; in the former structures the order topology is uniformizable, in the latter, compact. We conclude by showing that order convergence is not pretopological in the lattice $\mathscr{T}(S)$ of all topologies on an uncountable set $S$.

Let $L$ be a lattice, $x \in L, A \subset L$ and $\mathscr{F}$ a filter on $L$; then $x^{*}=\{y \mid y \geqq x\}$, $A^{*}=\{y \mid y \geqq x$, all $x$ in $A\}, \mathscr{F}^{*}=\cup\left\{F^{*} \mid F \in \mathscr{F}\right\}$. The symbols $x^{+}, A^{+}$ and $\mathscr{F}+$ designate the corresponding sets of lower bounds. A filter $\mathscr{F}$ is said to order converge to $x$ in $L$ if $x=\sup \mathscr{F}+=\inf \mathscr{F} *$. The order topology $\tau$ for $L$ is defined by taking those sets $A$ to be closed which contain all points to which filters that contain $A$ order converge.

An element $x$ in a lattice $L$ is compact if $x \leqq \sup A$ implies $x \leqq \sup A^{\prime}$, where $A^{\prime}$ is some finite subset of $A$. If each element in $L$ is the supremum of a set of compact elements, then $L$ is said to be compactly generated. Cocompact elements and cocompactly generated lattices are the dual notions. A lattice which is both compactly and cocompactly generated is said to be bicompactly generated.

Proposition 1. (a) Let $L$ be a lattice, $I$ a non-empty ideal, $D$ a non- 
empty dual ideal. Then the collection $\left\{a^{*} \cap b^{+} \mid a \in I, b \in D\right\}$ is an open subbase for a topology on $L$. We denote this topology by $\tau_{I D}$.

(b) If, in addition, $x=\sup \left(I \cap x^{+}\right)=\inf \left(D \cap x^{*}\right)$ for all $x$ in $L$, then $\tau_{I D}$ is a totally disconnected topology finer than the order topology for $L$.

(c) If $L$ is bicompactly generated, I the smallest ideal containing all compact elements, and $D$ the smallest dual ideal containing all cocompact elements, then $\left\{a^{*} \cap b^{+} \mid\right.$a compact, $\mathrm{b}$ cocompact $\}$ is a base for a topology on $L$ (call it $\tau_{c}$ ) and $\tau_{I D} \geqq \tau_{c}$.

Proof. (a) If $x \in L, a \in I, b \in D$, then note that $x \in(a \wedge x)^{*} \cap(b \vee x)^{+}$, and that this set belongs to the specified collection. Also note that the intersection of any pair of sets in the collection is again in the collection.

(b) Let $\mathscr{V}_{I D}(x)$ denote the neighborhood filter for $\tau_{I D}$ at $x$. Since $\left(\mathscr{V}_{I D}(x)\right)^{+}=I \cap x^{+}$and $\left(\mathscr{V}_{I D}(x)\right)^{*}=D \cap x^{*}$ it follows that $\mathscr{V}_{I D}(x)$ order converges to $x$, and hence $\tau$-converges to $x$. It is obvious that the sets of the open sub-base are closed in the order topology, hence closed in $\tau_{I D}$; since $\tau_{I D}$ separates any two points of $L$, it is totally disconnected.

(c) Upon observing that the set of compact elements is closed under finite joins, the argument becomes straight-forward.

THEOREM 1. (a) In a bicompactly generated lattice L, order convergence is topological, and the order topology is Hausdorff, totally disconnected and coincides with $\tau_{c}$.

(b) If, in addition, the set of compact elements is residual and the set of cocompact elements is antiresidual, then $\tau=\tau_{c}=\tau_{I D}$, where $\tau_{I D}$ is defined as in Proposition $1(\mathrm{c})$, and the lattice operations are continuous in the order topology.

Proof. (a) Let $\mathscr{V}_{c}(x)$ denote the $\tau_{c}$-neighborhood filter at $x$. It is easy to see that $\left(\mathscr{V}_{c}(x)\right)^{+}$contains all compact elements under $x,\left(\mathscr{V}_{c}(x)\right)^{*}$ contains all cocompact elements over $x$, and hence $x=\sup \left(\mathscr{V}_{c}(x)\right)^{+}=\inf \left(\mathscr{V}_{c}(x)\right)^{*}$. Thus $\mathscr{V}_{c}(x)$ order converges to $x$, for all $x$ in $L$, and $\tau_{c} \geqq \tau$.

For the reverse argument, we shall first establish that for each $x$ in $L$ the filter $\mathscr{V}(x)$ obtained by intersecting all filters that order converge to $x$ also order converges to $x$. Suppose that there is a compact element $c$ under $x$ which is not in $\mathscr{F}+$ for some filter $\mathscr{F}$ order converging to $x$. Then $c \leqq \sup \mathscr{F}^{+}=x$, and, since $c$ is compact, there are elements $y_{1}, \cdots, y_{n}$ in $\mathscr{F}+$ such that $c \leqq \sup \left\{y_{1}, \cdots, y_{n}\right\}$; furthermore for each $i=1, \cdots, n$, there is $F_{i} \in \mathscr{F}$ such that $y_{i} \in F_{i}^{+}$. Setting $F=\cap\left\{F_{i} \mid i=1, \cdots, n\right\}$, we have $c \in F^{+}$. Dually, every cocompact element over $x$ is in $\mathscr{F} *$. Thus if $\mathscr{F}$ order converges to $x$, then $\mathscr{F}$ contains every set $a^{*} \cap b^{+}$where $a \leqq x \leqq b$, $a$ compact, $b$ cocompact, that is $\mathscr{F} \geqq \mathscr{V}_{c}(x)$. Since the converse follows from the preceding paragraph it is clear that order convergence, since it coincides 
with convergence under the topology $\tau_{c}$, is topological, and $\tau=\tau_{c}$. A filter can order converge to at most one point; thus $\tau$ is Hausdorff. Finally, from Proposition 1 we deduce that $\tau$ is totally disconnected.

(b) If the compact elements of $L$ form a residual set, (i.e., $x$ compact and $y \leqq x$ implies $y$ compact) then they also form an ideal, and it is a simple matter to check that $L$ satisfies condition $c_{3}$ of [5]. It follows from part (a) of this theorem, that $L$ also satisfies condition $c_{1}$ of [5], and it follows immediately from Theorem 5, [5], that the lattice operations are continuous with respect to the order topology.

If $L$ is a lattice $L^{\prime} C L$ is a sub-complete lattice of $L$ if any non-void subset of $L^{\prime}$ has a supremum in $L^{\prime}$ which is also its supremum in $L$ and dually. The straight-forward proof of the next proposition will be omitted.

Proposition 2. A sub-complete lattice $L^{\prime}$ of a compactly generated lattice $L$ is again compactly generated. If $x$ in $L$ is compact, then $x_{c}=\inf \left\{y \in L^{\prime} \mid y \geqq x\right\}$ is a compact member of $L^{\prime}$, and $z \in L^{\prime}$ implies $z=\sup \left(z^{+} \cap\left\{x_{c} \mid x\right.\right.$ compact in $\left.\left.L\right\}\right)$.

THEOREM 2. (a) The order topology in an atomic Boolean algebra $L$ is uniformizable.

(b) A sub complete lattice $L^{\prime}$ of an atomic Boolean algebra $L$ is compact in its order topology.

Proof. (a) Let $I$ denote the smallest ideal containing all the atoms of $L$; let $D$ be the smallest dual ideal containing all the coatoms of $L$. ("Coatom" is the dual notion to atom; an atomic Boolean algebra is necessarily coatomic as well.) It is not hard to verify that $I$ is precisely the set of all compact members of $L, D$ the set of all cocompact members of $L$. Since each element $z$ in $L$ is the supremum of all atoms under $z$ (by Lemma $l$, p. 70, [4]), $L$ is bicompactly generated, and the order topology has the form described in Theorem 1.

For each $a \in I, b \in D$, let $V_{a b}=\left\{(x, y) \mid y \in(a \wedge x)^{*} \cap(b \vee x)^{+}\right\}$, and let $\beta=\left\{V_{a b} \mid a \in I, b \in D\right\}$. It is easy to see that $\beta$ is a filter base on $L \times L$ and that each member of $\beta$ contains the diagonal. If $(x, y) \in V_{a b}$ and $(y, z) \in V_{a b}$, then $a \wedge x \leqq y \leqq b \vee x$ and $a \wedge y \leqq z \leqq b \vee y$, which implies that $a \wedge x \leqq z \leqq b \vee x$; in other words, $V_{a b} \circ V_{a b} \subset V_{a b}$. Finally, let $x^{\prime}$ denote the complement of any element $x$ in $L$. Given $V_{a b}$ in $\beta$, let $c=b^{\prime}$, $d=a^{\prime}$. From the distributivity of $L$, we can deduce that $(x, y) \in V_{c d}$ if and only if $(y, x) \in V_{a b}$; hence $V_{a b}^{-1}=V_{c d}$ is a member of $\beta$. Thus $\beta$ generates a uniformity $\mathscr{U}$ on $L$; the uniform neighborhoods at a point $x$ in $L$ have the form $V_{a b}(x)=\{y \in L \mid a \wedge x \leqq y \leqq x \vee b\}$. But these are precisely the $\tau$-neighborhoods of $x$, and hence $\tau$ is the topology induced by the uniformity $\mathscr{U}$. We remark that another base for $\mathscr{U}$ is that consisting of all sets of the form $\{(x, y) \mid a \wedge x=a \wedge y\}$ for any $a$ in $I$. 
(b) Proposition 2, Theorem 1, and the first paragraph of the proof of Theorem 2a can be combined to show that the order topology on $L^{\prime}$ is the relative topology obtained from the order topology on $L$. Northam [6] has shown that the interval topology (which has a closed sub-base of sets of the form $a^{*}$ and $b^{+}$for arbitrary lattice elements $a$ and $b$ ) is Hausdorff in an atomic Boolean algebra. The interval topology on $L^{\prime}$ coincides with the relativized interval topology inherited from $L$, and hence is also Hausdorff. But A. J. Ward [7] showed that the order topology is compact in a complete lattice whenever the interval topology is Hausdorff, and so the theorem is proved.

It is not hard to verify that the lattice operations in $L$ are uniformly continuous with respect to the uniformity $\mathscr{U}$ described in the proof of Theorem 2a.

\section{2}

Before giving two examples which are relevant to our previous theorems, we will mention some examples from the literature. Kent [5] has constructed complete lattices in the Euclidean plane for which: (1) order convergence fails to be pretopological (order convergence is pretopological if the filter obtained by intersecting all filters order converging to $x$ also order converges to $x$ for each $x$ in the lattice); (2) order convergence is pretopological but not topological; (3) order convergence is topological, but the lattice operations are discontinuous in the order topology. Floyd [2] has given a classic example of a complete Boolean algebra whose order topology is not Hausdorff.

Our first example is very short and simple; it shows that the conclusion of Theorem $2 \mathrm{~b}$ cannot be extended to an arbitrary complete bicompactly generated lattice.

EXAMPLE 1. Let $L$ be a lattice consisting of an infinite collection of non-related elements with a least element 0 and greatest element 1 adjoined. $L$ is trivially complete and bicompactly generated. Note that the order topology for $L$ is discrete.

In the second example we show that order convergence is not pretopological in the lattice $\mathscr{T}(S)$ of all topologies on an uncountable set $S$. $\mathscr{T}(S)$ is compactly generated, atomic, and coatomic. If $I$ is the ideal of compact elements and $D$ the smallest dual ideal containing all of the coatoms, then it follows from a result by Fröhlich [3] that $\mathscr{T}(S)$ satisfies the conditions of Proposition 1b. This example demonstrates that one cannot greatly weaken the conditions assumed in Theorem la.

EXAMPLE 2. Let $S$ be the set of real numbers, $\mathscr{T}(S)$ the lattice of all 
topologies on $S$. Let $K$ be the collection of all topologies $p$ of the following form: $p$ has an open base consisting of a finite number of disjoint bounded "open" intervals with rational endpoints. Let $J$ be the collection of all topologies $q$ of the form: $q$ has an open base consisting of a finite number of disjoint bounded "open" intervals with irrational endpoints. Note that the indiscrete topology is the only member of $K \cap J$. Let $\tau$ be the "usual" topology on $S$, and let $\mathscr{F}_{1}$ and $\mathscr{F}_{2}$ be filters on $\mathscr{T}(S)$ defined as follows:

$\mathscr{F}_{1}$ is generated by sets of the form $p \cap \tau^{+}$, for $p \in K$;

$\mathscr{F}_{2}$ is generated by sets of the form $q \cap \tau^{+}$, for $q \in J$.

We have $\mathscr{F}_{1}^{*}=\mathscr{F}_{2}^{*}=\tau^{*}$; hence, inf $\mathscr{F}_{1}^{*}=\inf \mathscr{F}_{2}^{*}=\tau$. Also $\mathscr{F}_{1}^{+}=K$ and $\mathscr{F}_{2}^{+}=J$; the union of all topologies in $K$ (or in $J$ ) is a base for $\tau$; thus $\tau=\sup \mathscr{F}_{1}^{+}=\sup \mathscr{F}_{2}^{+}$. In other words, both $\mathscr{F}_{1}$ and $\mathscr{F}_{2}$ order converge to $\tau$. However, sup $(K \cap J) \neq \tau$, and Theorem 1, [5], tells us that order convergence is not pretopological in $\mathscr{T}(S)$.

The authors wish to express their thanks to the referee for his comments.

\section{References}

[1] R. P. Dilworth and P. Crawley, 'Decomposition theory for lattices without chain conditions', Trans. Amer. Math. Soc. 96 (1960), 1-22.

[2] E. E. Floyd, 'Boolean algebras with pathological order topologies', Pacific J. of Math. 5 (1955), 687-689.

[3] O. Frölich, 'Das Halbordnungssystem der topologischen Raume auf einer Menge', Math. Annalen 156 (1964), 79-95.

[4] P. R. Halmos, Lectures on Boolean Algebras (Van Nostrand Co. 1963)

[5] D. C. Kent, 'On the order topology in a lattice', Illinois J. of Math. 10 (1966), 90-96.

[6] E. S. Northam, 'The interval topology of a lattice', Proc. Amer. Math. Soc. 4 (1953), $824-827$.

[7] A. J. Ward, 'On relations between certain intrinsic topologies in partially ordered sets', Proc. Cambridge Philos. Soc. 51 (1955), 254-261.

Washington State University

Pullman, Washington

U.S.A. 